\title{
Study on the temperature field and thermal stress in the concrete box
}

\section{girder bridges}

\author{
Jian Shen ${ }^{1,}$, Xiaoyun Liü,b \\ ${ }^{1}$ School of Civil Engineering and Architecture, Jiangxi Science \& Technology Normal University, \\ Fenglin Avenue 605\#, Nanchang, Jiangxi P.R.China 330013 \\ ${ }^{2}$ School of foreign languages, Jiangxi Science \& Technology Normal University, Fenglin Avenue \\ 605\#, Nanchang, Jiangxi P.R.China 330013 \\ a894282069@qq.com, b505132338@qq.com
}

Key words: Variable cross-section box girder, temperature field, temperature stress

Abstract: Concrete bridge structures are usually exposed to the natural environment. Because of sunshine, wind, precipitation, etc natural environment influence, the concrete structure is changing temperature. With the poor thermal conductive performance of concrete, the upper structure temperature gradient appears while the temperature is changing. The existence of the nonlinear temperature field will lead to thermal stress in concrete bridge structure. The temperature of the variable cross-section box girder section has been observed for three months. Based on the actual observations, simplifying the system necessarily and reasonably and choosing the corresponding boundary conditions according to the project needs, the selected variable cross-section box girder section on temperature field is analyzed and calculated by using the finite element software ABAQUS.

\section{Introduction}

Bridge deck longitudinal temperature deformation, usually can be solved with the method of setting expansion joint. While cross the bridge, it often can't be dissipated temperature stress by using the method of direct setting expansion join because of integrity requirements. For stable cross section bridge, the temperature of the deformation and the temperature stress value of each section cross the bridge deck width is relatively small value and usually can be omitted. It is necessary for specialized research that the bridge with variable cross section, because each cross section deformation, will produce deformation constraint each other and result in the thermal stress which can not be ignored. In this paper, the research work is based on a certain variable cross-section of a highway ramp. The total box girder width is 32.8 63.2 meters and route project area belongs to subtropical monsoon climate.

\section{Scene temperature observation}

Considering the engineering local climate characteristics and the amplitude and average of daily temperature change, a total of 73 days on-site temperature observation is done. The box girder temperature difference at different positions is mainly decided by whether there is sunshine or shade. In order to save workload, the actual temperature observation can be measured on the temperature of the sunlight surfaces and no sunlight ones. Considering the specific situation, the temperature observation is done at 8:00, 11:00, 14:00, 17:00 and 20:00 everyday. The temperature 
of the observation point interval can be obtained by linear interpolation method. After 20:00, the night temperature changes more gently and the box girder structure slow releases heat absorbing during the day. The box girder temperature field distribution is uniform and the thermal stress is negligible. So the temperature observation can be stopped at 20:00. A box girder surface temperature observation is done at 5:00 and the temperature value of the box girder structure is regarded as the initial temperature of the structure unit thermal stress analysis.

\section{Temperature field finite element analysis}

The box girder sunshine thermal stress analysis is a process of dynamic change. The box girder temperature field and thermal stress field is changing with the change of external environment factors. The thermal stress field is determined by the temperature field distribution. The temperature field analysis and thermal stress analysis are often analyzed by different unit types in the finite element software. The Heat Transfer analysis unit and Heat Transfer analysis step are used in solving the temperature field distribution and the 3D Stress analysis unit and Static General analysis step are used in solving the thermal stress field. Considering the unit type and step analysis, the analysis process must have two steps. The first step is to solve the box girder internal temperature field distribution by using thermodynamics analyzing thermal dissipation unit, establishing model, inputting related materials corresponding thermodynamic parameters(such as specific heat, coefficient of thermal conductivity) and determining boundary conditions. After determine the temperature field, the restart function is used to obtain the thermal stress field by importing the box girder internal temperature field distribution into Predefined field as a model field variables, substituting the model mechanical parameters such as elastic modulus, poisson's ratio and constraint condition.

In the process of temperature field analysis, the DC3D8 (an 8 - node linear heat transfer brick) unit is adopted. Considering the box girder volume, the global unit size is set as 20 to reduce the calculation time in the preliminary analysis and calculation. A ccording to the calculation results, the analysis and calculation in detail is done at the temperature gradient large and the temperature field dramatic position by using Partition division command and generating more detailed structure grid. The material parameter selection of relevant physical quantities is shown as Table 1 . Although the thermal and mechanical properties of steel is completely different from concrete, but the role of the steel bar can be negligible in the whole process of thermal conductivity of box girder because of its negligible volume. The temperature field is shown as fig.1.

Table1 Box girder concrete main related values

\begin{tabular}{cccc}
\hline parameter & value & parameter & value \\
pyroconductivity & $2.55 \mathrm{~W} / \mathrm{m}^{\circ} \mathrm{C}$ & elasticity modulus & $3.45 \mathrm{E}^{4} \mathrm{MPa}$ \\
specific heat capacity & $930 \mathrm{~J}^{\circ} \mathrm{Kg}^{\circ} \mathrm{C}$ & poisson's ratio & 0.2 \\
thermal expansion coefficient & $1.0 \mathrm{E}^{-5}$ & density & $2400 \mathrm{~kg} / \mathrm{m}^{3}$ \\
\hline & &
\end{tabular}

Fig.1 Box girder section temperature field distribution nephogram (August 23rd, for example) 
The output data type must be set as some suitable form to offer restart analysis variable field for the subsequent process of temperature stress finite element analysis after the temperature field distribution calculation is finished. In addition to the default output ODB (the output database file) type, the file RES (restart file) type must be output to restart analysis. Configured at the main menu Step, the Output RES (restart file) file is exported by the Output function module to offer the temperature field calculation results for later analysis. Based on the box girder temperature field finite element analysis, two marked characteristics can be discovered.

1. Under the sunshine, the temperature field distribution inside the box girder structure is uneven and the temperature gradient near the roof surface is great, but with the increase of the depth, temperature gradient reduced sharply. The temperature gradient in box girder roof is approached zero. It is identical with the worse thermal conductive of concrete. The temperature gradient larger part is mainly appeared in the box girder roof surface to the deep about $2 \mathrm{~cm}$ below the surface of parts within a piece of thin layer structure. And the center of the box girder roof center about $30 \mathrm{~cm}$ thick, the temperature gradient is almost close to zero. Box girder roof map of tire temperature field result is shown in figure 2 .
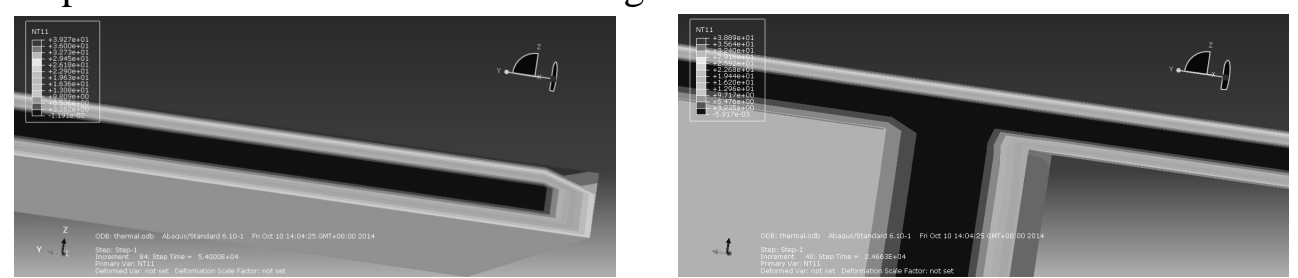

Fig.2 Box girder section temperature field distribution nephogram (14:00, August 23, for example)

2. When the roof and side wing plate is sunshine at the same time, there is a very significant linear thermal belt distribution on the edge of the wing. The heat agglomeration phenomenon will be appeared at the two surface junction. While the high-temperature belt area is very small and approximate to linear distribution, but it is likely to have larger thermal stress because of the heat accumulation. The box girder wing plate edge temperature field nephogram is shown as Fig.3.

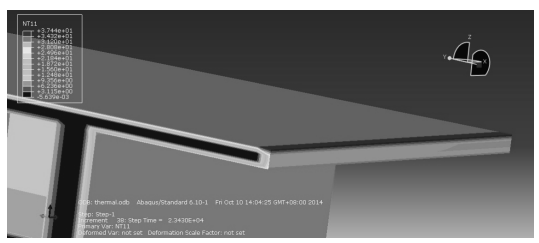

Fig.3 Box girder wing plate edge temperature field nephogram (14:00, August 23, for example)

\section{Thermal stress analysis}

The temperature field distribution is imported by the Restart function. The temperature field and thermal stress analysis is different analysis model. A new model can be build by Copy Model function and be reproduced analysis type from Heat Transfer into Static thermal analysis Model by the Replace function. The unit type is chosen as C3D8 (an 8 - node linear brick) and the unit size can be invariant.

The box girder temperature field distribution is set as a field variable. Opening the edit Field variables manager Field manager, the field state is changed from the default Propogated to Reset to initial and the concrete temperature Field distribution result is set as a new Field variable of thermal analysis.

A new thermal stress analysis job is restarted by setting the restart analysis in the Job editor (Edit Job) dialog box. After the operation, the box girder thermal stress nephogram and 
stress-time curve of each element node can be solved. The box girder section thermal stress nephogram(14:00, August 23, for example) is shown as Fig. 4.
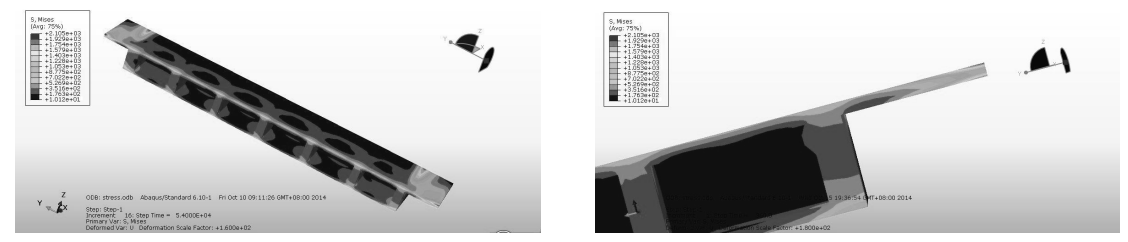

Fig. 4 box girder section thermal stress nephogram (14:00, August 23, for example)

\section{Conclusion}

The maximum thermal stress is mainly existed in box girder wing edges. Based on the box girder temperature field distribution analysis, the edge of box girder temperature is the highest and the thermal expansion deformation of box girder is difference at different locations on the edge of the wing. The thermal expansion deformation is constrained by each other position and the maximum thermal stress is produced.

The major values of thermal stress is appeared in the box girder roof and wall junction location. The box girder roof is suffered the most strong sunshine and with the maximum temperature gradient. The box girder wall can be regarded as a enclosure space with tinny temperature variations. The thermal expansion of box girder roof is subjected to restriction of box girder wall plate and the great thermal stress is generated.

The maximum temperature stress is on the edge of the box girder wing surface location and the depth, less than $1 \mathrm{~cm}$, is much thinner than the reinforced concrete protective layer thickness. So the effect of the steel bar inside the box girder for overcoming the concrete box girder structure surface thermal stress is very limited.

\section{Acknowledgments}

This research was supported by Dr. Scientific Research Startup Funds of Jiangxi Science \& Technology Normal University (3000990105), which is gratefully acknowledged.

\section{References:}

[1] Kennedy J. B,Soliman M.H. Temperature distribution in composite bridges[J]. Joumal of Struetural Engineering, ASCE, 1997, 113(3):475-482

[2] Zhang Yue, Hu Zhaotong, Jia Run-zhong. Temperature gradient of RC continuous curved box girder bridge [J]. Journal of Chang'an University(Natural Science Edition) , 2006 , 26(4):58-62

[3] Ariyawardena N, Ghali A, Elbadry M. ExPerimental Study on thermal cracking in reinforced conerete members[J]. Structural Journal . ACI. 1997, 94(4)

[4] Zhang Jianrong, Zhou Yuanqiang, LinJianping, Zhang Zhiyan. Solar Radiation Affection on Concrete BoxGirder Temperature Effect [J]. Journal of TONGJI university (NATURAL SCIENCE), 2008, Vol36(11):1479-1484

[5] Peng You-song, Zhu Xiaowen, Qiang Shizhong. Three Dimensional Analyses of Thermal Stresses in Concrete Box-girders [J]. JOURNAL OF THE CHINA RAILWAY SOCIETY. 2009,Vol39 (3):116-121 